This item was submitted to Loughborough's Research Repository by the author.

Items in Figshare are protected by copyright, with all rights reserved, unless otherwise indicated.

\title{
Electroluminescence imaging of PV devices: camera calibration and image correction
}

PLEASE CITE THE PUBLISHED VERSION

http://ieeexplore.ieee.org/servlet/opac?punumber=1000561

PUBLISHER

(C) IEEE

VERSION

AM (Accepted Manuscript)

\section{PUBLISHER STATEMENT}

This work is made available according to the conditions of the Creative Commons Attribution-NonCommercialNoDerivatives 4.0 International (CC BY-NC-ND 4.0) licence. Full details of this licence are available at: https://creativecommons.org/licenses/by-nc-nd/4.0/

\section{LICENCE}

CC BY-NC-ND 4.0

\section{REPOSITORY RECORD}

Bedrich, Karl G., Martin Bliss, Thomas R. Betts, and Ralph Gottschalg. 2019. "Electroluminescence Imaging of PV Devices: Camera Calibration and Image Correction". figshare. https://hdl.handle.net/2134/22893. 


\title{
Electroluminescence Imaging of PV Devices: Camera Calibration and Image Correction
}

\author{
Karl G. Bedrich, Martin Bliss, Thomas R. Betts, Ralph Gottschalg \\ Centre for Renewable Energy Systems Technology (CREST), \\ Loughborough University, Loughborough, Leicestershire, LE11 3TU, UK
}

\begin{abstract}
This paper presents an extensive image correction method for electroluminescence (EL) images of PV devices. This includes e.g. camera lens, single-time-effect, flat field, artefact and perspective correction. It demonstrates, that EL images, taken at different positions, can be normalized and prepared for quantitative analysis. Results show that after correction of images of the same module in different perspective positions, pixel position and intensity are practically identical. The paper also gives an overview of the software dataArtist. This is an open source software used for EL image processing, which has the presented correction and calibration routine implemented.
\end{abstract}

\section{INTRODUCTION}

Spatial resolved electroluminescence (EL) imaging of PV devices is a fast and easy applicable measurement method, first proposed by Fuyuki et al. [1]. Is was found suitable for qualitative analysis (e.g. detection of shunts, pre-breakdown sites, cracks, broken fingers and interconnectors) as well as quantitative analysis (diffusion length, local voltage, series resistance mapping) for cells [2-5] as well as modules [6, 7].

The analysis of EL images and similarly photoluminescence images requires dedicated and specialized software. For common image processing problems such as geometric measurements, filtering and edge detection imageJ, a Java based open source program, is often used [8-10]. Others have created own routines based on 'MATLAB' [11] or LabView $[12,13]$.

The scope of image processing on EL/PL images can be separated into the following problems:

- Images correction

- Automated detection of material/electrical defects (e.g. cracks) [14-17]

- Prediction of electrical properties e.g. localized series resistance and saturation current $[11,18,19]$

Programs incorporating these features can be also used to evaluate the state/quality of the PV device. Only few programs have their own graphical user interface (GUI) [15] and are commercially available [16, 19, 20].

However, for EL applications no software tool could be found dedicated to camera calibration and the removal of EL signal distortions, essential for quantitative analysis and interlab comparison. This was the motivation for creating an individual software solution, called dataArtist. This program aims to improve and standardize EL image analysis, providing an intuitive and powerful user interface (Section XII).

\section{IMAGE CORRECTION OVERVIEW}

To correct EL images, related distortions have to be removed. For this an image correction routine was implemented (Fig. 1). It uses two EL images as well as a dedicated camera calibration file. The routine sequence is chosen in order for distortions not to impair following steps.

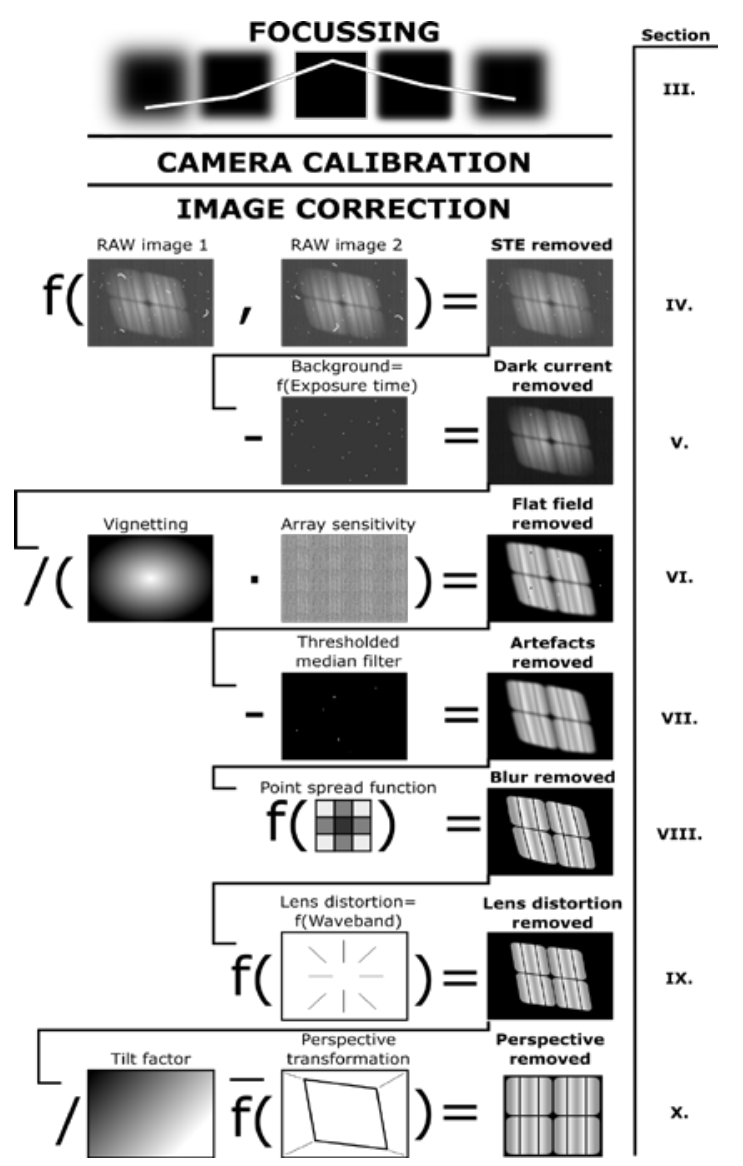

Fig. 1. Overview of the proposed image correction routine. Details to each of the steps are given in the sections shown on the right.

\section{BEST FOCUS DETERMINATION}

Many EL camera systems use a manual focus where the best sharpness is estimated through subjective comparison of images at different focus levels. In order to find the optimum 
focus a parameter qualifying the focus level can be helpful. Pertuz et al. compares various focus measure operators towards their robustness to noise, image contrast, saturation and window size for 3d reconstruction from a single image [21]. He concludes that Laplacian based operators have the best all over performance but also that the operator performance depends strongly on the imaging setup. A selection of four promising parameters was chosen from this source for application on EL images. Two different CCD cameras (A, B) and four different PV technologies were compared. The focus level was manually changed to obtain 9-13 focal points before and behind the image plane. The comparison (Fig. 2) shows that Tenengrad is the only parameter with the expected single maximum indicating best focus.
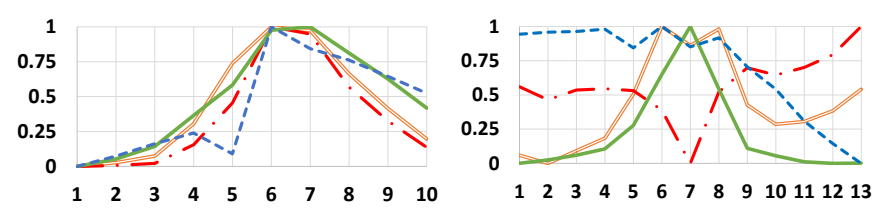

a) Cam. A, poly-Si module

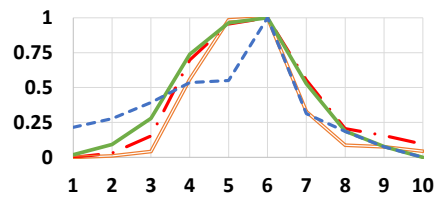

c) Cam. A, CIGS module

modified Laplacian

- Tenengrad

b) Cam. B, EFG mini module

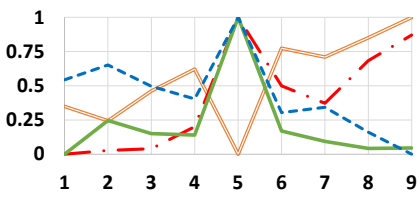

d) Cam. B, c-Si test cell

- variance of Laplacian

--- normalized gray-level variance

Fig. 2. Comparison of four relative focus parameters on two cameras and four PV technologies. All values normalized.

The trend of the absolute Tenengrad for all different focus levels (Fig. 2a) is exemplary shown in Fig. 3. The value range differs for the examined images and the chosen focus parameter. Therefore, these parameters cannot be used to calculate the absolute image sharpness. For this the point spread function (PSF) has to be measured as detailed in Section VIII.

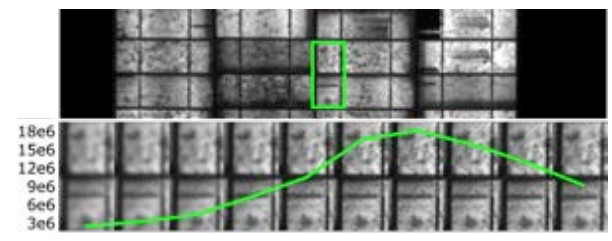

Fig. 3. EL image of a poly-Si module. Green box: area of image detail. Green plot: Absolute Tenengrad of the image detail. [22]

\section{SINGLE-TIME-EFFECT REMOVAL}

Single time effects (STE) are caused by cosmic high energy radiation interacting with the cameras CCD array. They can be seen as small spots or straight to curvy lines within an EL image. Their visibility increases with increasing exposure time and decreasing junction voltage of the device. Depending on their occurrence in EL or background image the spots will be brighter or darker than the EL signal. Especially in the latter case they can be easily confused with cell defects such as shunts. Because of their random distribution within images, their probability to occur twice at the same position is negligible. STE statistics and removal using a conditional minimum is detailed in [23].
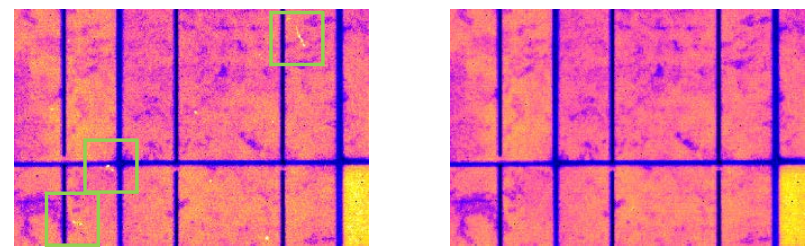

Fig. 4. EL image before (left) and after (right) STE removal. Some STE highlighted (green box) [23]

\section{DARK CURRENT REMOVAL}

Removal of environmental stray light and dark current (thermal noise and defective pixels) is often done through subtracting an EL image by an image of the same setup and exposure time under open circuit. [7, 13, 24-26]

As for EL images, these background images are prone to noise and STE decreasing the quality of EL images after subtraction. Both problems can be significantly reduced by taking at least two background images for different exposure times as input to solve $I_{B G}\left(\tau_{\text {exp }}\right)=a \cdot \tau_{\text {exp }}+b$. The resulting intercept $a$ and offset $b$ maps (Fig. 5) can be used to calculate the background image for a given exposure time $I_{B G}\left(\tau_{\text {exp }}\right)$ [27].

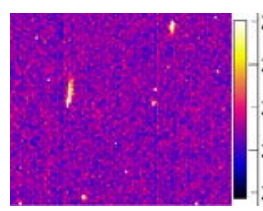

a) image (1/16)

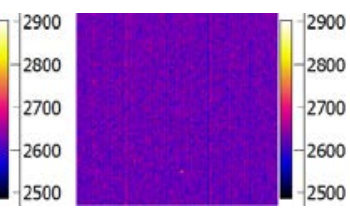

b) Fitted offset $b$

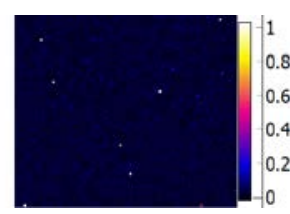

c) Fitted intercept $a$
Fig. 5: Excerpt of a dark current image at 600s exposure time

\section{FLAT FIELD REMOVAL}

Spatial non-uniformity in the camera sensor sensitivity originates from the inhomogeneous illumination of the sensor (vignetting) [10], sensitivity of the individual pixels and contamination of the optical system. It can be removed by dividing every taken EL image by a flat field image $I_{F F}$.

The methods to obtain $I_{F F}$ vary: A correction without an additional calibration image is proposed by Köntges et.al.: Here a named 'angle-of-view' calibration matrix was calculated from only the known aperture angle $\alpha_{a}$ of the camera [25]. Yet, this approach idealizes the actual flat field and ignores the individual pixel deviation. Other methods image a 'homogenous' light source at short distance and out of focus of 
the camera [7, 26]. It was reported that even a high resolution LCD flat panel emitting red light at $612 \mathrm{~nm}$ can be sufficient for measuring CdTe solar cells with a recombination peak at around $850 \mathrm{~nm}$ [28]. This method might come in handy but ignores that a LED flat panels or LCD displays by no means are homogeneous light sources. Hence, this paper proposes a simple, jet effective method to measure the flat field image $I_{F F}$ :

Similar to [28] this method images a red screen (Fig. 6), but takes at minimum 15 images of the screen at various positions and different rotation angles relative to the optical axis of the lens.
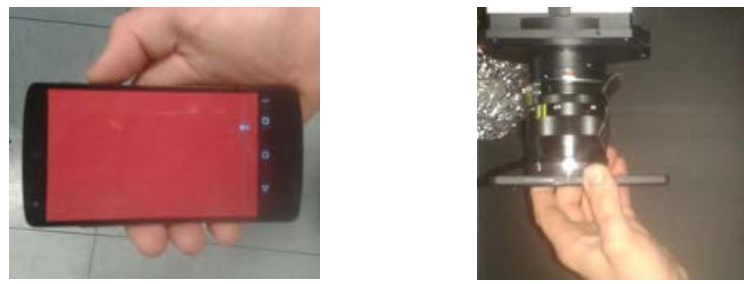

a) Red screen using mobile phone b) Screen placed on camera lens

Fig. 6. Setup for flat field calibration

For the bare eye the used screen looks homogenous. However, an intensity difference up to $\pm 10 \%$ was shown in a difference image from two identical looking images (Fig. 7b). An unintentionally introduced gap between screen and lens can also alter the result (Fig. 7c).

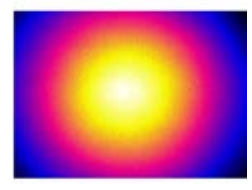

a)

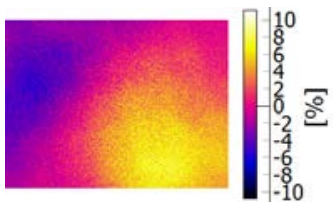

b) Difference image

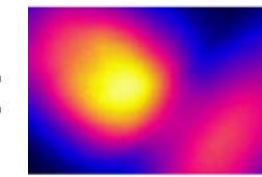

c) Result for titled screen
Fig. 7. Left: example images used for flat field calibration taken with setup (Fig. 6)

To take screen inhomogeneities and failed measurements into account to proposed method uses a conditional average of all taken images as follows:

- Remove dark current for the respective exposure time:

$$
I_{d, i}=I_{E L, i}-I_{B G}
$$

- $\quad$ To exclude faulty image areas, create mask $M_{i}$ for every $I_{d, i}$ selecting only areas higher than the successive created image average $I_{A v g}$, reduced by local noise level, defined by the noise level function (NLF) [27]:

$$
\begin{gathered}
M_{i}=I_{d, i}>\left[I_{A v g}-\operatorname{NLF}\left(I_{A v g}\right) \cdot 3\right] \\
I_{A v g}=\frac{\sum_{i=0}^{n} I_{d, i}\left[M_{i}\right]}{n}
\end{gathered}
$$

- $\quad$ Scale image average [0-1]:

$$
I_{F F}=\frac{I_{A v g}}{\max \left(I_{A v g}\right)}
$$

As the results in Section XI show, this method is suitable to remove flat field deviations. However, due to the limitations of the flat field being measured in a different waveband (red vs. near infra-red) and also not in measurement plane, a residual error remains. Additional methods to bypass these limitations will be covered in another publication.

\section{ARTEFACT REMOVAL}

Although dark current and flat field removal corrects most defective pixels, depending on the calibration date new pixel defects may remain. Their selection using a spatial standard deviation is described in [24, 25]. However, a higher stability towards outliers can be reached using a threshold median [13]. For this purpose a median filtered image $I_{\text {median }}$ is created. All pixels with a relative deviation to the given image $I$ higher than a threshold $T$ are set to the median filtered value:

$$
I\left[T<\frac{I-I_{\text {median }}}{I_{\text {median }}}\right]=I_{\text {median }}
$$

A pure median filter is sensitive to image features. For high $T$ however only high gradient deviations are filtered without deceiving the image quality. The filtered result is shown in Fig. 8.
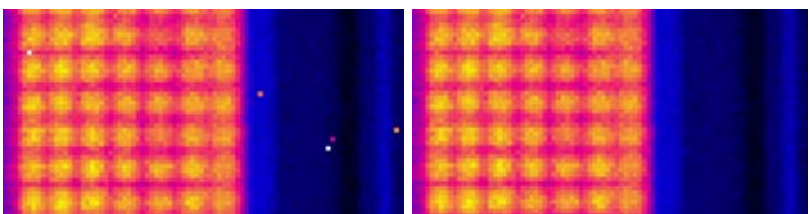

Fig. 8. EL image detail of a m-Si module before (left) and after artefact removal (right). Median kernel-size $=3 ; T=0.2$

\section{IMAGE DECONVOLUTION USING A POINT SPREAD FUNCTION}

EL and PL images occur blurred due to the focal mismatch, diffraction, chromatic aberration, photon scattering within the CCD sensor, light trapping and electrical smearing. Image deconvolution using a point spread function (PSF) can be used to increase the image contrast.

The PSF can be measured directly through imaging a point light source (e.g. gas lamp behind aperture in a dark room) [29]. However, due to the wavelength dependency of the PSF, its measurement should be within the luminescence waveband.

Walter, Teal and Breitenstein et al. determine the PSF as a transformation of a measured edge spread function, taken from a wafer edge or a masked area on top of a wafer [30-32]. This measurement neglects radial variation of the PSF but calculates the point spread even for higher distances (up to 500 pixels) from the edge. For module scale EL images, dataArtist implements a method for direct PSF measurement: detecting multiple pinholes of the size smaller than the image resolution. A Gaussian distribution is fitted in order to determine the center of each PSF used to average all PSF over each other. Fig. 14 
(left) shows the detected pinholes. In this example 147 detected light spots where used to create the PSF. The pinholes were created with a needle piercing into black flocked self-adhesive paper which was then taped on a glass plate.

\section{LENS DISTORTION REMOVAL}

Camera lens distortion can be described as a combination of radial ('barrel') and tangential distortion. These distortions can be extracted from the deviation of detected corner positions of a calibration (e.g. chessboard) pattern (Fig. 9) to an ideal grid. The implemented image correction uses the camera calibration of the $\mathrm{C}++$ framework OpenCV [33]. To reduce the influence of corner detection errors and inhomogeneities in the pattern, it is recommended to create at least 15 images of the pattern within image plane at different positions and orientation at least within the image. The size of the pattern should be within 25$75 \%$ of the image area.

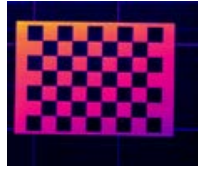

a) Chessboard

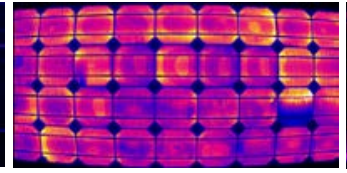

b) before correction

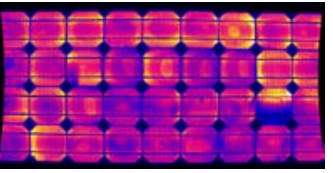

c) after correction
Fig. 9. EL image of a PV module before (b) and after removal of lens distortion (c). Distortions have been exaggerated for clarification [34]

\section{Perspective CORRECTION}

The alignment of corners of PV device and image is essential for image comparison within and across different institutions. The needed transformation- and shear matrix can be either obtained using a template image (pattern recognition), or through manual or automated corner detection. A tilt map, weighting the luminous intensity can be calculated from rotation and tilt angle, obtained from the device corners. [34]

For automated corner detection the following algorithm was found to be reliable:

- Create binary image $I_{b n}$ using the statistical EL signal minimum $E L_{\text {min }}: I_{b n}=I_{E L}>E L_{\text {min }}$

- $\quad$ Filter small features

- $\quad$ From each side: detect position of first positive pixel

- From all detected position: remove outlier and execute linear regression (Fig. 10)

- $\quad$ Refine line position via $\max \left(\operatorname{sum}\left(\frac{d I_{E L}}{d x}[\right.\right.$ line $\left.\left.]\right)\right)$

- $\quad$ Set device edges as intersection of fitted lines

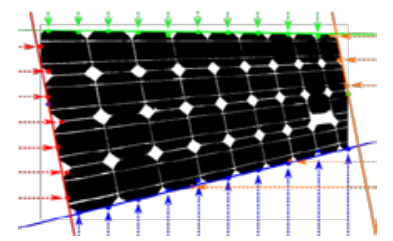

Fig. 10. Scheme of device corner detection

\section{REsUlts AND DisCUSSION}

The proposed image correction routine is evaluated using a mono-Si PV module imaged at four different positions:
a. No tilt, position 1
b. No tilt, position 2
c. $15^{\circ}$ tilt angle, position 1
d. $35^{\circ}$ tilt angle, position 1 , rotated

All images where captured in the large scale EL chamber 'HuLC' (CREST) using a SensoCam HR-830 camera with an image resolution of $3324 \times 2504$. All correction methods where executed automatically. Since the EL signal itself remains constant during imaging, a successful image correction leads to practically identical results.

Fig. 11 shows EL images with identical colour scheme and scale of all four positions before (left) and after image correction (right). Looking and e.g. the cells $(1,5)$ or $(2,3)$ (row, column) it is clear that the intensity due to vignetting and tilt factor differs. However, after image correction, differences in position and intensity are invisible. For position (c) and (d) corners of the image are missing because they were not imaged in the first place.

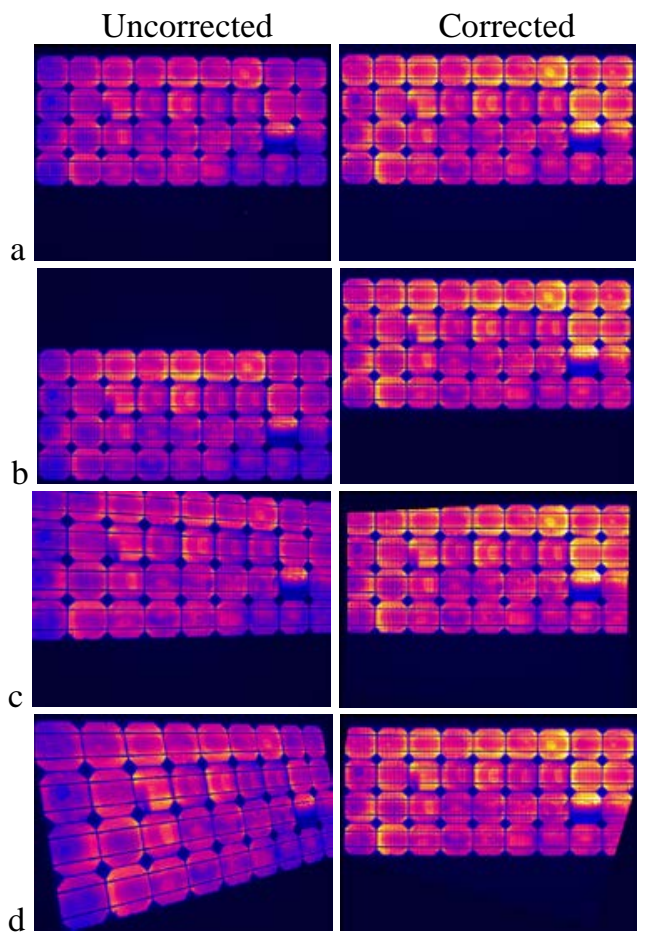

Fig. 11. Comparison of EL images before and after correction. Scale and colour scheme are identical for all images.

Fig. 12 details the individual difference from corrected positions (b-d) to (a) as a colour layer on top of image (a). The red box within the overview marks the position of the image detail. The majority of the image remains grey scaled. This indicates low and homogenous image differences.

A red arrow points on the reddish region below a crack in the image detail. It indicates that even transient EL instabilities can 
be recovered although the imaged position is different. The blue colouring around some cells indicates a positional error of $\sim 2$ pixel for position (d). This could be due a small bending or the module at a higher angle or due to a lens calibration being conducted under visible light resulting in a positional offset in some areas of the image.
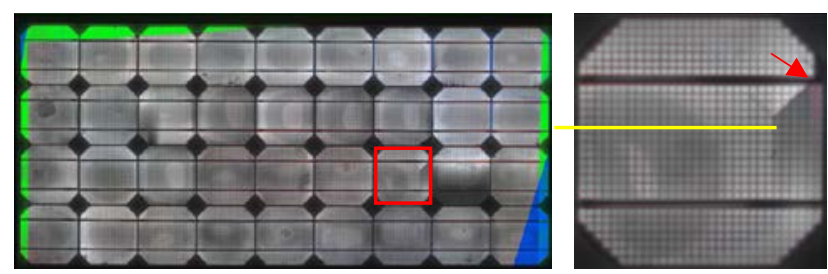

Fig. 12. EL comparison: overview (left) and detail (right). Differences to (a) visualized as colorlayer: b:red; c:green; d:blue; Transparency scaled between $0-50 \%$ relative difference

The yellow line in Fig. 13 shows the position of a line plot shown in Fig. 12 (yellow line). With a coefficient of variation of about $1 \%$ one can see that the cell intensity and location of all corrected positions (a-d) is practically identical.

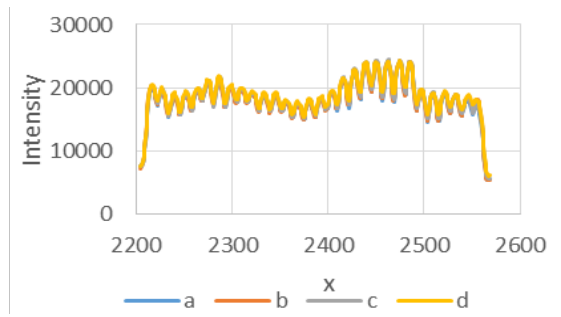

Fig. 13. Line plot of yellow line in Fig. 12

\section{IMPLEMENTATION IN DATAARTIST}

dataArtist includes several tools for camera calibration and image correction including the proposed methods in this paper. For camera calibration all needed images are dropped into dataArtist and the matching tool is executed. The determination of the point spread function is exemplary shown in Fig. 14. The calibration results are hereinafter saved to a calibration file.

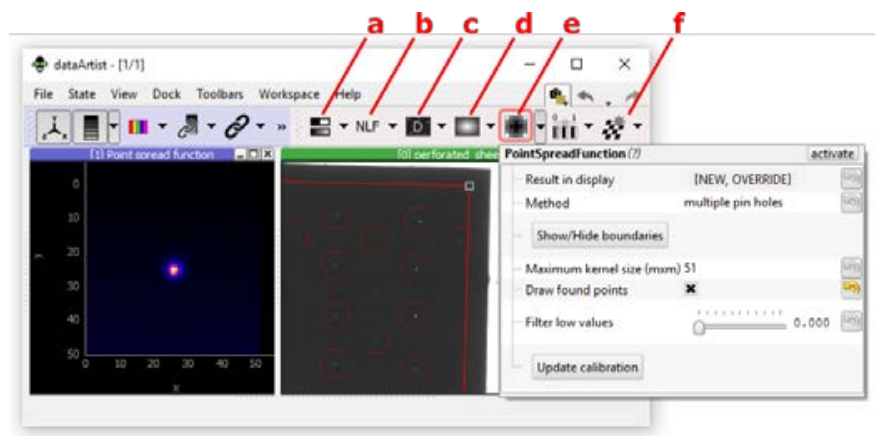

Fig. 14. dataArtist screenshot - toolbar 'calibration'. a: Best focus determination; b: noise-level-function measurement; c: Dark current mapping; d: Flat field mapping; e: PSF estimation (selected): f: lens distortion measurement
The correction of EL images using that calibration is shown in Fig. 15. Perspective correction (Fig. 15, red box) can be done either using the outline of the PV device (automatically detected or manually defined) or using a reference image.

The program and further instructions on the calibration and correction routine can be found at https://github.com/radjkarl/dataArtist.

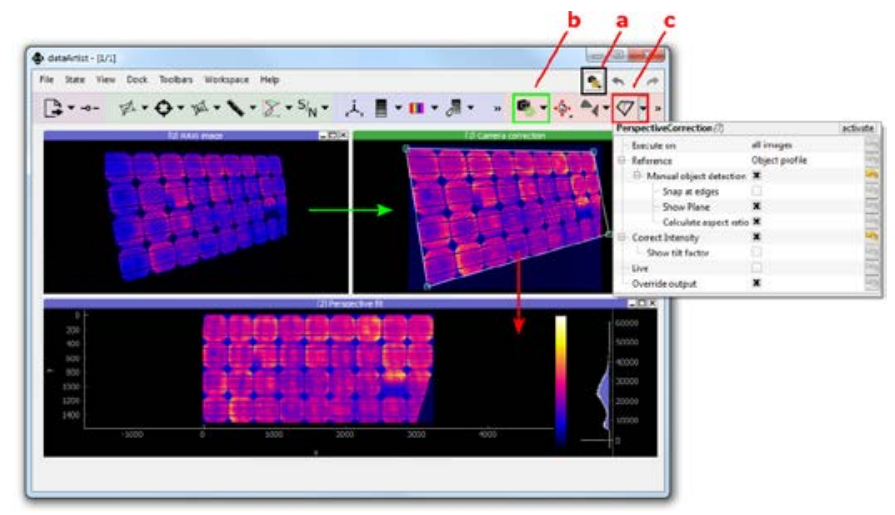

Fig. 15. dataArtist screenshot - Image correction step 1-5 using 'CorrectCamera' tool (green box and arrow); step 6 using 'PerspectiveCorrection' tool (red box and arrow); bottom: corrected image

\section{SUMMARY}

This paper proposes an extensive image and perspective correction and demonstrates that EL images of modules, taken at different positions and angles can be normalized. For the examined case of a $4 \times 9$ cell PV module at different positions and angles a positional error up to $2 \%$ and intensity difference about $1 \%$ was reached. This allows quantitative comparison of EL images taken in different perspectives or in different institutions. It builds the foundation for feature extraction from differently aged devices and for EL image comparison of the first EL round robin ongoing. All procedures are released under open source and can be executed within dataArtist, a free and powerful GUI dedicated to EL imaging.

\section{REFERENCES}

[1] T. Fuyuki, H. Kondo, T. Yamazaki, Y. Takahashi, and Y. Uraoka, "Photographic surveying of minority carrier diffusion length in polycrystalline silicon solar cells by electroluminescence,” Appl. Phys. Lett., vol. 86, no. 26, p. 262108, 2005.

[2] K. Bothe and D. Hinken, Quantitative luminescence characterization of crystalline silicon solar cells, 1st ed., vol. 89. Elsevier Inc., 2013.

[3] M. Glatthaar, J. Haunschild, R. Zeidler, M. Demant, J. Greulich, B. Michl, W. Warta, S. Rein, and R. Preu, "Evaluating luminescence based voltage images of silicon solar cells,” J. Appl. Phys., vol. 108, no. 1, p. 014501, 2010.

[4] T. Trupke, E. Pink, R. a. Bardos, and M. D. Abbott, "Spatially resolved series resistance of silicon solar cells 
obtained from luminescence imaging,” Appl. Phys. Lett., vol. 90, no. 9, p. 093506, 2007.

[5] P. Würfel, T. Trupke, T. Puzzer, E. Schäffer, W. Warta, and S. W. Glunz, "Diffusion lengths of silicon solar cells from luminescence images,” J. Appl. Phys., vol. 101, no. 12, p. 123110, 2007.

[6] B. Li, A. Stokes, and D. M. J. Doble, "Evaluation of twodimensional electrical properties of photovoltaic modules using bias-dependent electroluminescence,” Prog.

Photovoltaics Res. Appl., vol. 20, no. 8, pp. 936-944, 2012.

[7] T. Potthoff, K. Bothe, U. Eitner, D. Hinken, and M.

Köntges, "Detection of the voltage distribution in photovoltaic modules by electroluminescence imaging," Prog. Photovoltaics Res. Appl., vol. 18, no. 2, pp. 100-106, Mar. 2010.

[8] K. Zaunbrecher, "Imaging as Characterization Techniques for for Thin-Film Cadmium Telluride Photovoltaics,” Color. State Univ., vol. Dissertati, 2014.

[9] J. L. Crozier, "Characterization of cell mismatch in photovoltaic modules using electroluminescence and associated electro- optic techniques Magister Scientiae,” 2012.

[10] M. Bokalič, J. Raguse, J. R. Sites, and M. Topič, “Analysis of electroluminescence images in small-area circular CdTe solar cells,” J. Appl. Phys., vol. 114, no. 12, p. 123102, 2013.

[11] A. R. L. M. Ezquer Mayo, J.M. Cuadra, J. Díaz, J. Moracho, "New Analytical Software for Electroluminescence Results Out of Patterns," in 27th European Photovoltaic Solar Energy Conference and Exhibition, 2012, pp. 3041-3048.

[12] D. Kendig, G. B. Alers, and A. Shakouri, "Characterization of defects in photovoltaics using thermoreflectance and electroluminescence imaging," in Conference Record of the IEEE Photovoltaic Specialists Conference, 2010, pp. 17331736.

[13] M. Bliss, X. Wu, K. Bedrich, T. R. Betts, and R. Gottschalg, "Spatially and Spectrally Resolved Electroluminescence Measurement System for PV Characterisation," in IET-RPG, accepted 2015.

[14] A. Mansouri, M. Zettl, O. Mayer, M. Lynass, M. Bucher, and O. Stern, "Defect detection in photovoltaic modules using electroluminescence imaging," 27th Eur. Photovolt. Sol. Energy Conf. Exhib., vol. 64617926, pp. 3374-3378, 2012.

[15] D.-M. Tsai, S.-C. Wu, and W.-Y. Chiu, "Defect Detection in Solar Modules Using ICA Basis Images,” IEEE Trans. Ind. Informatics, vol. 9, no. 1, pp. 122-131, Feb. 2013.

[16] M. Solutions, "Electroluminescence Module Inspection Why Electroluminescence Imaging ?" 2013.

[17] S. Spataru, P. Hacke, D. Sera, S. Glick, T. Kerekes, and R. Teodorescu, "Quantifying solar cell cracks in photovoltaic modules by electroluminescence imaging," in 2015 IEEE 42nd Photovoltaic Specialist Conference (PVSC), 2015, pp. $1-6$.

[18] B. Mitchell, J. W. Weber, D. Walter, D. MacDonald, and T. Trupke, "On the method of photoluminescence spectral intensity ratio imaging of silicon bricks: Advances and limitations,” J. Appl. Phys., vol. 112, no. 6, 2012.

[19] M. P. I. of M. Physics, "Description Electroluminescence ( EL ) Imaging Evaluation Software.” Halle, 2012.

[20] greateyes GmbH, "LumiSolarCell System: Photo- and Electroluminescence Inspection Equipment for Wafers and Solar Cells.” Berlin, 2012.

[21] S. Pertuz, D. Puig, and M. A. Garcia, "Analysis of focus measure operators for shape-from-focus," Pattern Recognit., vol. 46, no. 5, pp. 1415-1432, May 2013.

[22] IEC, "IEC TS 60904-13 PHOTOVOLTAIC DEVICES Part 13: Electroluminescence of photovoltaic modules draft.” 2015.

[23] K. G. Bedrich, M. Bliss, T. R. Betts, and R. Gottschalg, "ELECTROLUMINESCENCE IMAGING OF PV DEVICES : SINGLE-TIME-EFFECT STATISTICS AND REMOVAL,” in PVSAT-12, 2016, no. 1, pp. 37-40.

[24] F. J. J.L. Crozier, E.E. van Dyk, "High Resolution Spatial Electroluminescence Imaging of Photovoltaic Modules," Vor. Nelson Mand. Metrop. Univ., 2009.

[25] M. Köntges, M. Siebert, and D. Hinken, “Quantitative analysis of PV-modules by electroluminescence images for quality control,” Proc. 24th ..., 2009.

[26] D. Hinken, K. Ramspeck, K. Bothe, B. Fischer, and R. Brendel, "Series resistance imaging of solar cells by voltage dependent electroluminescence,” Appl. Phys. Lett., vol. 91, no. 18, p. 182104, 2007.

[27] K. G. Bedrich, M. Bliss, T. R. Betts, and R. Gottschalg, "Electroluminescence Imaging of PV devices: Determining the Signal-To-Noise Ratio," 2016.

[28] M. Topič, J. Raguse, K. Zaunbrecher, M. Bokalič, and J. R. Sites, "ELECTROLUMINESCENCE OF THIN FILM SOLAR CELLS AND PV MODULES - CAMERA CALIBRATION," in 26th European Photovoltaic Solar Energy Conference and Exhibition, 2011, pp. 2963-2966. C. J. Schuler, M. Hirsch, S. Harmeling, and B. Sch, "NONSTATIONARY CORRECTION OF OPTICAL Nonstationary Correction of Optical Aberrations Anonymous ICCV submission,” ICCV - 13th Int. Conf. Comput. Vis., no. 1, 2011.

[30] O. Breitenstein, F. Fruhauf, and A. Teal, “An Improved Method to Measure the Point Spread Function of Cameras Used for Electro- and Photoluminescence Imaging of Silicon Solar Cells,” IEEE J. Photovoltaics, vol. 60, no. 5, pp. 1-6, 2016.

[31] D. Walter, A. Liu, and E. Franklin, "Contrast enhancement of luminescence images via point-spread deconvolution,”... (PVSC), 2012 38th ..., pp. 307-312, 2012.

[32] A. Teal and M. Juhl, "Correcting the inherent distortion in luminescence images of silicon solar cells," in IEEE PVSC, 2015.

[33] G. Bradski and A. Kaehler, Learning OpenCV: Computer vision with the OpenCV library. O’Reilly, 2008.

[34] K. G. Bedrich, M. Bliss, T. R. Betts, and R. Gottschalg, "Electroluminescence Imaging of PV Devices: Uncertainty due to Optical and Perspective Distortion,” in EU PVSEC, 2015, vol. 31, pp. $1748-1752$. 